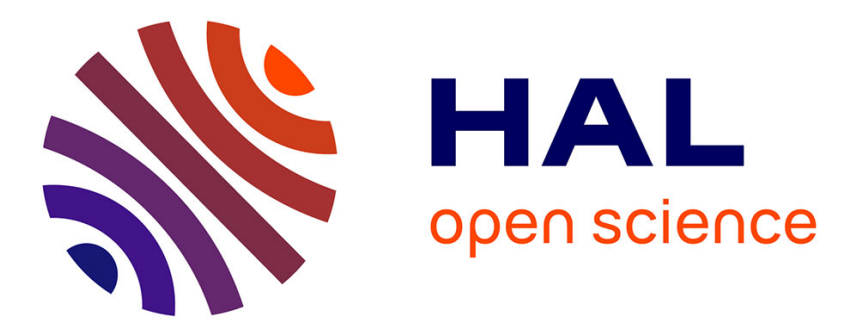

\title{
By-pass transition in microchannels under EDL effect
}

Sedat F. Tardu

\section{To cite this version:}

Sedat F. Tardu. By-pass transition in microchannels under EDL effect. International Conference on MEMS, NANO and Smart Systems (ICMENS), Aug 2004, Alberta, Canada. pp.293-400, 10.1109/ICMENS.2004.1508982 . hal-00262257

\section{HAL Id: hal-00262257 https://hal.science/hal-00262257}

Submitted on 27 Feb 2020

HAL is a multi-disciplinary open access archive for the deposit and dissemination of scientific research documents, whether they are published or not. The documents may come from teaching and research institutions in France or abroad, or from public or private research centers.
L'archive ouverte pluridisciplinaire HAL, est destinée au dépôt et à la diffusion de documents scientifiques de niveau recherche, publiés ou non, émanant des établissements d'enseignement et de recherche français ou étrangers, des laboratoires publics ou privés. 


\title{
By-pass transition in microchannels under the EDL effect
}

\author{
Sedat TARDU \\ Laboratoire des Ecoulements Géophysiques et Industriels \\ LEGI BP 53 X Grenoble Cedex-France \\ Sedat.Tardu@hmg.inpg.fr
}

\begin{abstract}
The effect of the electric double layer (EDL) on the bypass transition mechanism in the linear evolution stage is explored through direct numerical simulations. An initial perturbation velocity field consisting of a pair of counterrotating vortices is introduced in Poiseuille and EDL flows and the time-space evolution of the perturbed field is analysed for short times at half the critical Reynolds numbers (3000 for Poiseuille and 300 for EDL). The wall normal and spanwise perturbation velocities development are both quantitatively and qualitatively similar in macro and micro flows. The streamwise velocity, which is initially zero and set up by the generation of the wall normal vorticity is twice larger under the EDL effect. Both flows develop inclined strong streamwise shear layers. Overall is the close similarity of the disturbance evolution showing that the three dimensional linear mechanism in EDL flow lead to the structures that are at least as strong as in Poiseuille flow.
\end{abstract}

\section{Introduction and background}

We have recently shown that the electric double layer destabilizes considerably the linear instability of the micro-channel flows (Tardu, $2004 \mathrm{a}$ and b). It is recalled that the velocity profile under the electrokinetic EDL effect can be put in non-dimensional form as: $u=1-y^{2}$

$$
-4 \frac{I_{1}-I_{2}}{\frac{\kappa^{2} \sinh \kappa}{\bar{\zeta}^{2} G}+4\left(I_{3}-\frac{I_{4}}{\sinh \kappa}\right)}\left\{1-\left|\frac{\sinh \kappa y}{\sinh \kappa}\right|\right\}
$$

where the scaling velocity is the centreline velocity of the Poiseuille component, i.e. $-\frac{a^{2} d p / d x}{2 \mu}$ and the scaling length is the half channel height $a$. There are several parameters in this equation, for instance $G=\frac{\left(n_{0} z e a\right)^{2}}{\lambda_{0} \mu}$, with $n_{0}$ standing for the ionic number concentration, $z$ for the valence of positive or negative ions, $e$ for the electron charge $\lambda_{0}$ the electric conductivity of the fluid, and $\mu$ for its dynamic viscosity. One of the most important quantities involving in (1) is the non-dimensional DebyeHückle parameter

$$
\kappa=a k=a\left(2 n_{0} z^{2} e^{2} / \varepsilon \varepsilon_{0} k_{b} T\right)^{1 / 2}
$$

with $\varepsilon$ and $\varepsilon_{0}$ being respectively the dielectric constant of the medium and the permittivity of vacuum, $k_{b}$ the Boltzmann constant and $T$ the absolute temperature. The characteristic EDL thickness is $1 / k$. The non-dimensional Zeta potential reads for $\bar{\zeta}=\frac{z e \zeta}{k_{b} T}$. The quantities $I$ in (1) are given by:

$$
\begin{aligned}
& I_{1}=I_{3}=\frac{\cosh \kappa-1}{\kappa}, \\
& I_{2}=\left(\frac{1}{\kappa}+\frac{2}{\kappa^{3}}\right) \cosh \kappa-\frac{2}{\kappa^{2}} \sinh \kappa-\frac{2}{\kappa^{3}}, \\
& I_{4}=\frac{\sinh \kappa \cosh \kappa}{2 \kappa}-\frac{1}{2}
\end{aligned}
$$

The important difference between the EDL and macro-Poiseuille flow profiles is the presence of an inflexional point at $y \approx \frac{1}{\kappa} \arcsin h\left\{-\frac{2}{r \kappa^{2}} \sinh (\kappa)\right\}$ in the EDL profile where $r$ is the ratio of the EDL and Poiseuille flows centreline velocities. This makes the flow inviscidly unstable, according to the Fjortoft's criteria. The neutral curves deduced from the hydrodynamic stability analysis are summarized in Fig.1. It is clearly seen that the critical Reynolds number decreases by $a$ factor nearly equal to 2 under the EDL effect at $\kappa=41$ : the critical wave and Reynolds numbers of the microflow are respectively $\alpha_{c}=1.10$ and $R e_{c}=3190$, to be compared with $\alpha_{c}=1.02$ and $R e_{c}=5772$ of the conventional Poiseuille flow. This effect can be more appreciated if it is recalled that, at $\kappa=41$, the friction factor increases by only some $10 \%$. It is clear that one of the most significant effects of EDL is the decrease of the critical Reynolds number, rather than the increase in friction coefficient or the apparent viscosity. 
To each couple $\left(\kappa, G \bar{\zeta}^{2}\right)$ corresponds a different set of the critical wave and Reynolds numbers $\left(\alpha_{c}, R e_{c}\right)$. It is difficult to illustrate the interdependency of $R e_{c}$ via $G \bar{\zeta}^{2}$, since $\alpha_{c}$ changes continuously as $G \bar{\zeta}^{2}$ varies. We fixed the wave number at its macro-scale flow critical value $\alpha_{c}=1.02$ to give a general tendency and extract clear information.

There is a range of $G_{\bar{\zeta}}^{2}$ in which the critical Reynolds number decreases sharply before reaching a plateau region wherein $R e_{c}$ is close to the critical number deduced from the neutral curves. This range varies with $\kappa$ and it is about two decades large. The lower and upper limits of the $G \bar{\zeta}^{2}$ range increase with $\kappa$. It is clear both from Fig. 2 that $\operatorname{Re}_{c}$ is more sensitive to $G \bar{\zeta}^{2}$ when the Debye-Hückel parameter is small enough, say for $\kappa \leq 40$. The first and main condition to expect significant EDL effects on hydrodynamic linear stability is to deal with liquids of low ionic concentration. The second condition requires large enough $G \bar{\zeta}^{2}$ value. This is fulfilled by low conductivity, which is generally associated with wall/liquids interactions that lead to high Zeta potential $\bar{\zeta}$. The second condition is less critical than the first. To show this, consider a median value of $\kappa=20$.

The non linear saturation of the primary stability and formation of a secondary flow, together with the secondary instability processes have to be analyzed in EDL flow similarly to the Poiseuille macro-flow. Some arguments on the reinforcing effect of the EDL on the subcritical nature of the macro Poiseuille flow may however already be given. The square of the amplitude of a finite disturbance is given by:

$$
\frac{d\left|A_{1}^{2}\right|}{d t}=2 \alpha c_{i}\left|A_{1}\right|^{2}+\left(k_{1}+k_{2}+k_{3}\right)\left|A_{1}\right|^{4}
$$

according to Stuart (1960). The flow reaches a subcritical equilibrium state when $k_{1}+k_{2}+k_{3}>0$. The coefficient $k_{1}$ represents the distortion of the mean motion: it is related to the eigenfunctions of the linear stability problem, and it is negative. The coefficient $k_{2}$ is linked to the generation of the harmonic of the fundamental and is also likely negative. The wall normal distortion of the fundamental $\left(k_{3}\right)$ must " be positive and outweigh the combined negative effect of $k_{1}$ and $k_{2}$ to reach a subcritical state". Now, $k_{1}$ is proportional to $\operatorname{Re}_{c} \alpha_{c}^{2}$. It has therefore a significantly smaller negative contribution to $k_{1}+k_{2}+k_{3}$ under the EDL effect. Furthermore, part of the terms involving in the coefficient $k_{3}$ is inversely proportional to $\mathrm{Re}_{c}$ and the EDL presumably reinforces the positive character of $k_{3}$ in the subcritical state.
The EDL effect on the nonlinear stability mechanism is analyzed through the spatio-temporal development of a localized disturbance in a channel flow by Direct Numerical Simulations in this investigation. A perturbation related to a pair of counter rotating vortices is followed in time and space with and without EDL. The main aim is to investigate the effect of the double electrostatic layer on the linear, nonlinear and breakdown stages in the by-pass transition process.

There are at least two fundamental reasons to investigate the by-pass transition in micro-channel flows. This transition scenario is related to the disturbance growth on time scales significantly shorter than typical TollmienSchilichting (TS) waves that "by-passes" the spatial and temporal development of the two-dimensional disturbances and their inherent secondary instabilities. The set-up of three-dimensionality leads to the achievement of finite amplitudes and of the non-linear effects. They can mainly be generated by local surface irregularities such as the roughness. The latter, is of course not a micro-effect, because it affects the characteristics in macro and micro flows exactly in the same manner at similar scaled roughness dimensions. Yet, it is more difficult to control the roughness geometry and dimensions in microchannels. Note that the roughness does not affect the stability characteristics of a developed internal flow, in the TS waves sense, except in the entrance region. Thus, the bypass transition process is a presumably more common phenomena in micro-flows. The second reason to investigate the development of the $3 \mathrm{D}$ localized disturbances has to the associated with the rapid disturbance algebraic growth. The two-dimensional waves are always the least stable modes according to the Squire's theorem. However, the damping of oblique waves may be smaller at subcritical Reynolds numbers as already shown and mentioned in several investigations (see Henningson et al., 1993 for example). The present study deals with the detailed analysis of the time-space development of a localized disturbance in wall bounded flows with inflexional (EDL) mean velocity profiles compared to the non-inflexional (Poiseuille) ones. Because of the large computational efforts needed, only small amplitude disturbances in their early stage of development could be analyzed in this paper. More results including medium and large amplitude disturbances leading to the formation of turbulent spots shall be presented in the Symposium.

\section{Direct Numerical Simulations (DNS)}

The Orlandi's (2001) DNS code has been used and modified for the present purpose. The fractional step is used with the non-linear terms explicitly treated by a Runge-Kutta scheme. The viscous terms are discretized in time with the Crank-Nicholson scheme. A nonuniform grid in the wall normal direction is applied through a stretched coordinate transformation. The set of equations is integrated by a fractional step method through which 
imposed streamwise and spanwise periodicity permit the use of Fourier series. The computations are performed on a IBM pSeries 630 (Power 4, $1.45 \mathrm{GHz}$ ) workstation and at IDRIS (Computational Center of the CNRS-French National Research Center). The dimensions of the computational domain are $2 a$ in the wall normal direction, $16 \pi a$ in the streamwise $x$, and $8 \pi a$ in the spanwise $z$ directions. The quantities are scaled by the half channel height and the centerline velocity of the related Poiseuille flow ( $\left.-\frac{a^{2} d p / d x}{2 \mu}\right)$. Thus, the time scale for instance is $-\frac{2 \mu}{a d p / d x}$. The number of modes is $256 \times 128 \times 128$ in the $x, y$ and $z$ directions. The resolution for the Poiseuille flow is better than Henningson et al. (1993) for the small perturbation amplitude case.

\section{Localized initial disturbance}

The initial structure is two pairs of counter-rotating vortices exactly as in Henningson et al. (1993). The form is:

$$
\psi=\varepsilon f(y)\left(x^{\prime} / l_{x}\right) z^{\prime} \exp \left[-\left(x^{\prime} / l_{x}\right)^{2}-\left(z^{\prime} / l_{z}\right)^{2}\right]
$$

and the velocity components of the perturbed field are:

$$
\left(u^{\prime}, v^{\prime}, w^{\prime}\right)=\left(-\psi_{y} \sin \vartheta, \psi_{z^{\prime}},-\psi_{y} \cos \vartheta\right)
$$

in respectively the streamwise, wall normal and spanwise directions. The lengths $l_{x}$ and $l_{z}$ stand for the streamwise and spanwise lengths of the disturbance $\left(l_{x}=l_{z}=2\right.$ ) and $\varepsilon$ is its amplitude. The angle $\vartheta$ of the disturbance can be used as a parameter governing the distribution of the initial energy in different regions of wavenumber space. One took:

$$
f(y)=(1+y)^{p}(1-y)^{q}
$$

with $p=q=2$, as in Henningson et al. (1993). The perturbations (5) are superimposed to the mean velocity profile as initial conditions, and the flow field is followed in time and space. The angle of the perturbation is $\vartheta=0$ here. Thus the initial streamwise velocity perturbation component is null.

The instantaneous 3D non-dimensional Navier Stokes equation under the EDL external force:

$$
\frac{\partial u_{i}}{\partial t}+\frac{\partial u_{i} u_{j}}{\partial x_{j}}=-\frac{\partial p}{\partial x_{i}}+\frac{1}{\operatorname{Re}} \frac{\partial^{2} u_{i}}{\partial x_{j}^{2}}-\frac{r \kappa^{2}}{\operatorname{Re}} \frac{|\sinh \kappa y|}{\sinh \kappa}
$$

is adequately resolved in time and space with the previously indicated initial conditions. The last forcing term is of course absent in the macro-scale Poiseuille simulations. The streaming current is the weighted integral of the charge density by the cross-sectional velocity field:

$$
I_{S}=\int u(x, y, z, t) \rho(x, y, z, t) d S=S<u \rho>
$$

where $<>$ stands for the cross-section average and $S$ is the cross-sectional area of the channel. The streaming current equals to the conduction current in the steady-state configuration. Denoting the mean and the fluctuating quantities respectively by $\bar{q}$ and $q^{\prime}$, one has $I_{S}=S<\bar{u} \bar{\rho}>+S<u^{\prime} \bar{\rho}>+S<\bar{u} \rho^{\prime}>+S<u^{\prime} \rho^{\prime}>\quad$ with $\bar{u}=\bar{u}(y)$ and $\bar{\rho}=\bar{\rho}(y)$ for a $2 \mathrm{D}$ channel flow. The first term is the steady state value and results in the electric force given as the last term in the equation (7). The second term is negligible when $\frac{\sqrt{u^{\prime} u^{\prime}}}{\bar{u}}<<1$, which is the case in the stability analysis conducted here. The set-up of the charge density fluctuations $\rho^{\prime}$ depends on whether the Boltzmann distribution is valid or not. The left hand side of the Nernst-Planck equation governing the ionic concentration $n_{i}$ can be put in the form:

$\nabla^{2} \bar{n}_{i}-P e_{x} \frac{\partial \bar{n}_{i}}{\partial x}-P e_{y} \frac{\partial \bar{n}_{i}}{\partial y}--P e_{z} \frac{\partial \bar{n}_{i}}{\partial z}+\nabla \cdot\left(\frac{z_{j} e \bar{n}_{i}}{k_{b} T} \nabla \xi\right)$

where $\xi$ is the electric potential, and $P e$ is the Péclet number with:

$P e_{x}=\left(\bar{u}+u^{\prime}\right) \frac{a}{D_{i}}, P e_{y}=v^{\prime} \frac{a}{D_{i}}, P e_{z}=w^{\prime} \frac{a}{D_{i}}$

$D_{i}$ standing for the diffusion coefficient of the type-i ion. The largest unsteady term is clearly $P e_{y} \frac{\partial \bar{n}_{i}}{\partial y}$ which is still negligible because of the small amplitude $v^{\prime}$ fluctuations. The time dependent terms of the convective-diffusion Nernst-Planck equation are therefore small in the present investigation, and the electric force is adequately modeled in the Eq. 7 , providing that the velocity profile $\bar{u}=\bar{u}(y)$ is not appreciably affected by the secondary instability.

\section{Results}

There are several factors that make the computations substantially heavy and time consuming. First of all, the EDL layer has to contain at least 10 points or so. This is hard to achieve even in the stretched coordinate system when the Debye-Hückle parameter is large. The computational domain has to be increased to obtain a selfsimilar form of the spot and the number of modes has also to be large enough. That is particularly important when the amplitude of the initial perturbation is large, which is, on the other hand necessary to describe the non-linear stages of the transition process. All the computations are performed in double precision that is time consuming at least in the data processing stage. For these reasons, we are 
able to present only partial results here corresponding to the early linear stages of the development of a small amplitude localized disturbance corresponding to $\varepsilon=0.0001$ and a maximum initial normal velocity amplitude of $4 \cdot 10^{-6}$. A flow with a relatively severe EDL effect is chosen for the purpose and for the same reasons. We took $\kappa=10, G=764$ and $\bar{\zeta}=0.89$ that would typically correspond to a flow in a microchannel of $25 \mu \mathrm{m}$ height, of an infinitely diluted $\mathrm{KCl}$ aqueous solution $\left(n_{0}=3.764 \times 10^{19} \mathrm{~m}^{-3}\right)$ subjected to a Zeta potential of 22 $\mathrm{mV}$. The Debye length is $1.25 \mu \mathrm{m}$, and the bulk conductivity is $\lambda_{0}=8 \times 10^{-9} \mathrm{~S} / \mathrm{m}$. The critical Reynolds number in this case is 635 compared with 5772 of the macro flow. Thus, since the aim is to analyze the subcritical nature of the by-pass mechanism, we took $R e=3000$ in the Poiseuille flow and $R e=300$ in the EDL flow, i.e. ten times smaller than in the macro-flow.

Fig. 2 shows the spatial evolution of $v^{\prime}$ component of the perturbation velocity at $y=-0.56$ and $t=10$ in Poiseuille and EDL flows. Please be aware that the maximum and minimum of the velocity fields and the contour spacing are not identical in the figures. We tried to choose these parameters to have a reasonable "topological similarity" between micro and macro flows, and they differ because of the differences in the base flows and the Reynolds numbers. The reader may first compare the results corresponding to the Poiseuille flow with those of Henningson et al. (1993, their Fig. 2, p. 181) to notice the excellent correspondence, despite the differences used in the numerical parameters and procedures. We see the characteristics of the dispersion of a wave packet with streamwise elongation and spanwise inclination of the velocity contours. Globally the time and space evolution of the wall normal and spanwise perturbation velocities are both qualitatively and quantitatively similar in the macro and micro-flows (remember that the EDL Reynolds number is ten times smaller). However, the spanwise gradient of $v^{\prime}$ is significantly more important in EDL than the Poiseuille flow pointing at a relative enhancement of the streamwise vorticity (not shown here).

One of the basic feature of the linear transient (or algebraic) growth mechanism is the set-up of a streamwise velocity field while, it is initially zero (since $\vartheta=0$ ). The algebraic growth is related to the generation of the wall normal vorticity $\omega^{\prime}{ }_{y}=-\frac{\partial w^{\prime}}{\partial x}+\frac{\partial u^{\prime}}{\partial z}$ by the tilting of the mean spanwise vorticity through the $\frac{\partial u}{\partial y} \frac{\partial^{\prime}}{\partial z}$ source term. A second plausible explanation can be based on arguments similar to the evolution of the transport equations in turbulent shear flows. The temporal and spatial evolution of some local average $\left\langle u^{\prime} v^{\prime}\right\rangle$ is produced by $-<v^{\prime} v^{\prime}>\frac{\partial u}{\partial y}$, that, in return generates $<u^{\prime} u^{\prime}>$ through the $-2<u^{\prime} v^{\prime}>\frac{\partial u}{\partial y}$ term. The $u^{\prime}$ algebraic growth is an order of magnitude larger than $v^{\prime}$ and $w^{\prime}$ in both flows.

One of the aims of this investigation is to explore the control possibilities of micro-scale flows through the EDL effects. It is asked whether the transition can be controlled by transient application of an external electric force. A numerical experiment has been conducted to have preliminary insight into these aspects. The external force is applied at $\mathrm{t}=0$ and shut down immediately after, i.e. the force term in the equation (4) was $-\frac{r \kappa^{2}}{R e} \frac{|\sinh \kappa y|}{\sinh \kappa} \delta(t)$, $\delta(t)$ standing for the Dirac function. The experiments have shown the profound transient effect of the EDL field. The flow feature at short times is quite similar to the flow under the continuous EDL forcing with the apparition of an inclined streamwise shear layer at $t=20$, as in the macroflow (Fig.3). This is a common characteristic of the threedimensional linear mechanism (Breuer and Haritonidis, 1990; Henningson et al., 1993). The shear layer is also present in the transient EDL flow at $t=10$, with lesser steepness (not shown here).

In a general manner, the Fourier components that have a potential of exponential growth such as associated with inflectional instabilities will unlikely experience large transient growth except when the initial condition has a sufficient projection on the two-dimensional wave space. We, therefore tried an axisymmetric disturbance with zero normal vorticity:

$$
\begin{aligned}
& \left(u^{\prime}, v^{\prime}, w^{\prime}\right)=\left(-\frac{x}{r^{2}} \psi_{y}, \frac{1}{r} \psi_{r},-\frac{z}{r^{2}} \psi_{y}\right) \\
& \psi=\frac{\varepsilon}{2} f(y) r^{2} \exp \left(-\left(\frac{r}{l}\right)^{2}\right)
\end{aligned}
$$

with $r^{2}=x^{2}+z^{2}, l=10, \varepsilon=0.4$ and $f(y)$ same as in (6) with $p=2, q=5$. This disturbance is qualitatively close to the flow induced by an axisymmetric jet at the lower wall of the channel. The perturbation took rapidly the form of a small turbulent spot with the clear apparition of the small scales as it is seen in Fig. 4.

\section{Conclusion}

The electric double layer severely destabilizes in the linear sense the macro Poiseuille flow providing that the liquid contains a small number of ions and that the fluidsurface interaction leads to large enough Zeta potential. The Tollmien-Schilichting waves have their own secondary instabilities and the non-linear saturation characteristics. The by-pass transition mechanism is different, in the sense that the disturbance growth on time scales significantly shorter than the TS waves. The local disturbances may be of different forms and caused by several factors such as surface roughness. 
The time-space development of such a disturbance set-up by two counterrotating vortices has been analyzed here, in an EDL flow with a non-dimensional Debye-Hückel parameter equal to ten. The results are systematically compared with the macro-scale Poiseuille flow. Only the perturbation with small amplitude could be reported here. The results show that the perturbation velocity components behave in a quite similar manner in EDL and Poiseuille flows, when the scaling is based on the centerline velocity and the channel half-width. The basic feature of the threedimensional linear stability scenario is the generation (or reinforcement) of wall normal vorticity created by the tilting of the mean spanwise vorticity. This mechanism leads to the set-up of zones of large streamwise velocity variations and the formation of internal streamwise shear layers. The latter are accentuated by EDL, at least in the early stages of transient growth.

The problem contains a large number of parameters and the computations are time consuming. Yet, our main aim is to study the medium and large amplitude perturbations leading to turbulent spots. The main question, for instance, is the determination of subcritical transitional Reynolds numbers related to localized disturbances. These points are currently being investigated and more results shall be presented at the symposium.

The experimental verification of both the linear and nonlinear stability mechanism under the EDL effect is difficult as we largely discussed before (Tardu, 2004 a and b). The EDL effect has a sense when the liquid contains a small amount of ions and the Zeta potential is large enough. For instance, the theoretical value of the Debye length in the pure water is about one micron, but it is difficult to eliminate all the impurities especially in the double layer. Other liquid/surface configurations would certainly be more convenient for experimental purposes. One could for instance use some hydrocarbures combined with additives in metallic channels. Such liquids have been largely investigated in the literature for a while for the electrisation problems. The Debye length in a cyclohexane $\left(\mathrm{C}_{6} \mathrm{H}_{12}\right)$ flow containing the tri isoamylammonium picrate is for example as large as $40 \mu \mathrm{m}$ (Andriamitanjo, p. 51) and the Zeta potential is $\zeta=45 \mathrm{mV}$. The transitionstability experiments can be conducted in minichannels of height larger than $800 \mu \mathrm{m}$ with such liquids, and large Reynolds number can be reached without any specific difficulties.

\section{Acknowledgments}

We warmly thank IDRIS (Institut du Développement et des Ressources en Informatique Scientifique) of CNRS (Centre National de Recherche Scientifique) for its continuos support.

\section{References}

[1] Andriamitanjo S. "Electrisation d'un hydrocarbure s'écoulant dans un tube métallique en régime laminaire" Thèse de l'INPG, 1981, Grenoble, France.

[2] Breuer, K.S., Haritonidis, J.H., 1990 The evolution of a localized disturbance in a laminar boundary layer. Part 1. Weak disturbances, J. Fluid Mech. ,220, 359-391.

[3] Breuer, K.S., Landahl, M.T., 1990 The evolution of a localized disturbance in a laminar boundary layer. Part 2. Strong disturbances, J. Fluid Mech. ,220, 595-621.

[4] Henningson D.S., Lundbladh A., Johansson A., 1993 A mechanism for bypass transition from localized disturbances in wall-bounded shear flows, J. Fluid Mech., 250, 169-207.

[5] Orlandi P., 2001 Fluid Flow Phenomena, A numerical Toolkit, Kluwer Academic Publishers, Dordrecht-Boston-London.

[6] Stuart J.T., 1960 "On the non-linear mechanics of wave disturbances in stable and unstable parallel flows. Part 1 The basic behaviour in plane Poiseuille flow" J. Fluid Mech., 9, pp. $353-370$

[7] Tardu S., 2004-a Interfacial Electrokinetic Effect on the Microchannel Flow Linear Stability, J. Fluid Eng. , 126, pp. 1013, 2004.

[7] Tardu S., 2004-b Analysis of the electric double layer effect on the microchannel flow stability. To appear in Microscale Thermophysical Engineering. 


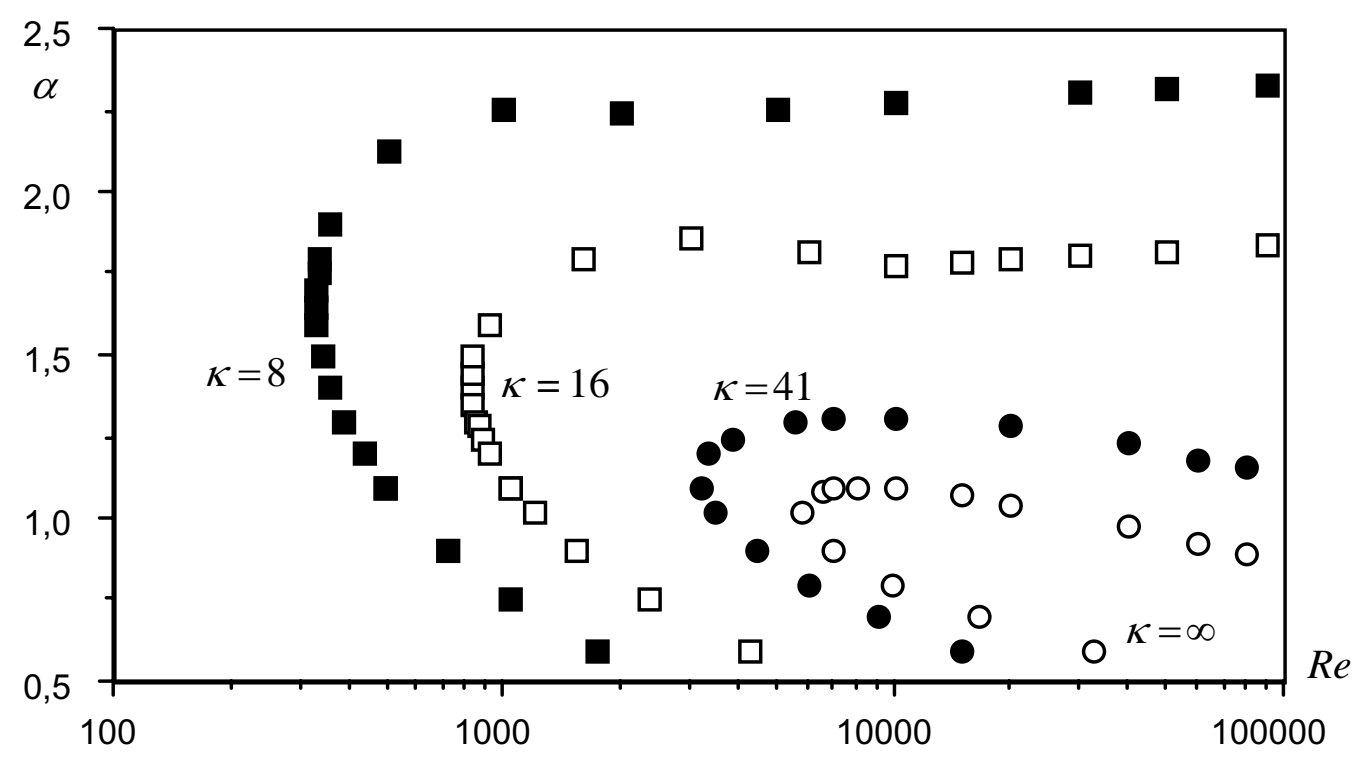

Figure 1 Neutral curves of the EDL flow compared with the Poiseuille flow. a) The open circles correspond to Poiseuille flow with $\kappa=\infty$. Bold circles correspond to $\kappa=41, G=12720$ and $\bar{\zeta}=2.1254$ ( reference case). The rest of the results are obtained by changing the microchannel height and keeping constant the rest of the parameters. The triangle is obtained for $\kappa=164$ b) Neutral curves for $\kappa=8,16$ and 41 compared with the macroscale flow.
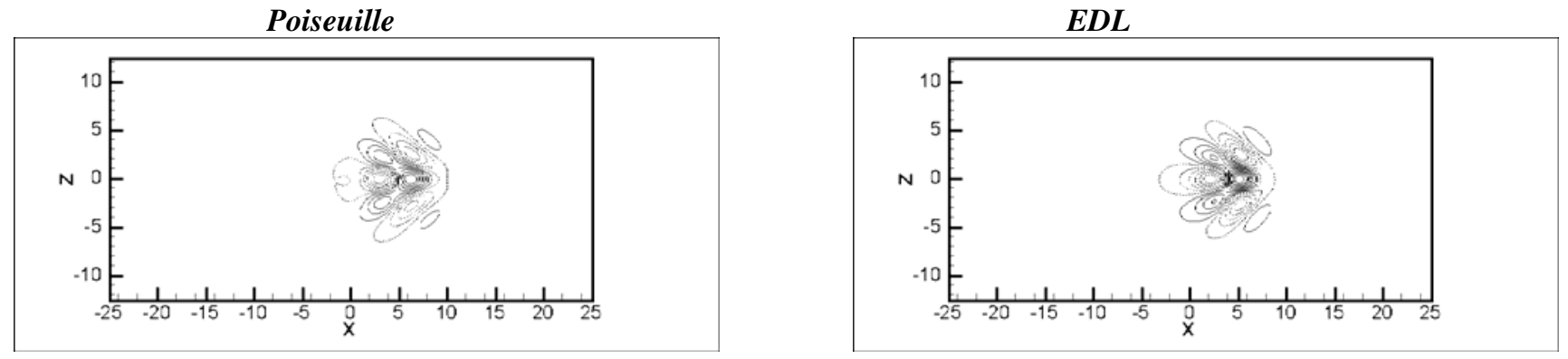

Figure 2 The wall normal perturbation velocity contours for Poiseuille $(R e=3000)$ and EDL flows $(R e=300)$ at $t=10$ and $y=-0.56$. The maximum and minimum velocities in Poiseuille flow are $\pm 5 \times 10^{-6}$, and in EDL flow $\pm 4 \times 10^{-6}$. 
Poiseuille

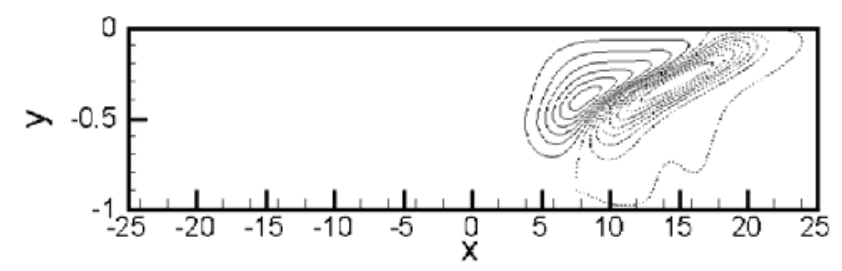

$E D L$

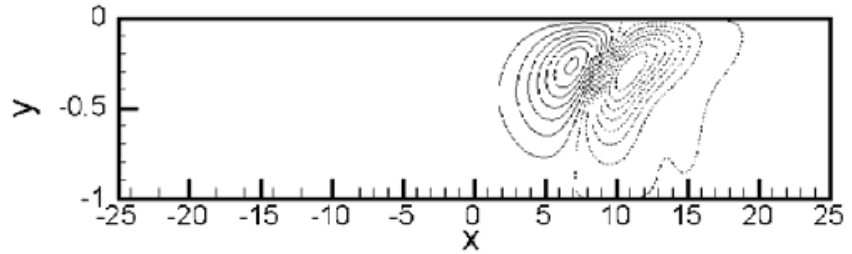

Figure 3 The streamwise perturbation velocity contours for Poiseuille $(R e=3000)$ and EDL flows $(R e=300)$ at $t=20$ in the $x-y$ plane at $z=0$, when the electrical force field is turned off at $t=0$ (see the text). The maximum and minimum velocities in Poiseuille flow are $\pm 10 \times 10^{-5}$, and in EDL flow $\pm 6 \times 10^{-5}$.

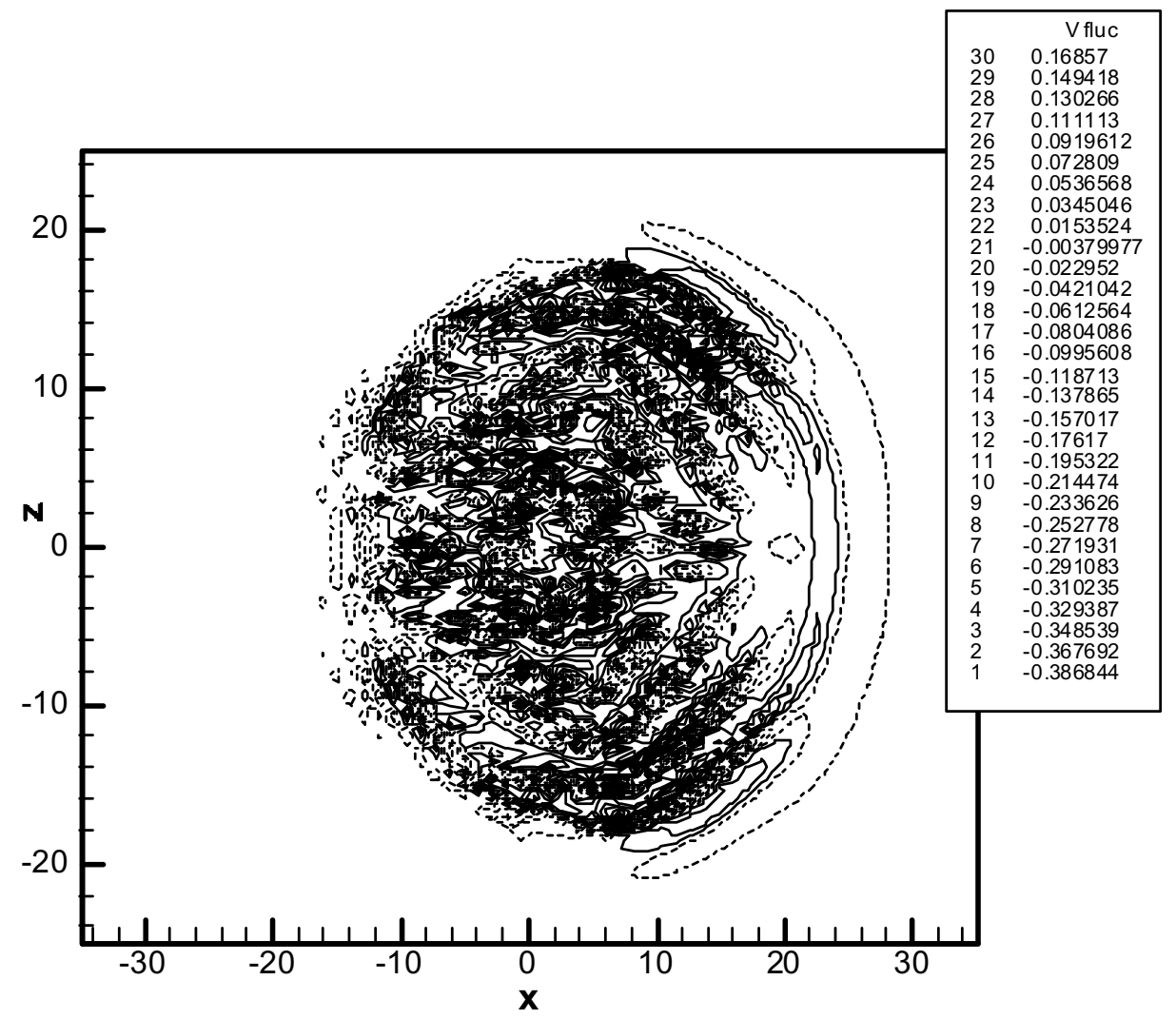

Figure 4 The wall normal perturbation velocity contours the EDL flows $(R e=300)$ at $t=15$ in the $x-z$ plane at $y=-0.8$ with an axisymmetric disturbance. Note the apparition of small scales in the turbulent spot. 